\title{
Eye-tracking aplicado a recursos pedagógicos visuais e multimídia: um mapeamento sistemático
}

\author{
Vítor Felicio Salema, Carla Lopes Rodriguez, João Ricardo Sato \\ Centro de Matemática, Computação e Cognição - Universidade Federal do ABC - Santo \\ André, SP - Brasil \\ vitor.salema@aluno.ufabc.edu.br, c.rodriguez@ufabc.edu.br, \\ joao.sato@ufabc.edu.br
}

\begin{abstract}
Eye-tracking is an eyeball screening technique that has been used for the benefit of several areas, among them, the educational area. In this sense, a systematic mapping study was conducted in order to investigate which visual pedagogical resources are explored with this technique and the physiological and/or cognitive aspects considered in this analysis between the years 2010 and 2019, so as to get an overview of the current research scenario and contribute with future studies. The results point a lack of studies with instructional videos, slideshows and affectivity, in contrast to a large number of studies on texts and attention.
\end{abstract}

Resumo. Eye-tracking é uma técnica de rastreio do globo ocular que vem sendo utilizada em beneficio de diversas áreas, entre elas a da educação. Nesse sentido, um mapeamento sistemático de literatura foi conduzido com o objetivo de investigar quais recursos pedagógicos visuais são explorados com essa técnica, e os aspectos fisiológicos elou cognitivos considerados nessas análises entre os anos de 2010 e 2019, para, assim, obter-se um panorama do atual cenário de pesquisa a fim de contribuir com estudos futuros. Os resultados apontam para uma carência de pesquisas com vídeos instrucionais, apresentações de slides e afetividade no contexto educacional, em contraste com um grande número de estudos analisando o recurso texto e a atenção.

\section{Contextualização}

Dados obtidos a partir da técnica de rastreio ocular e de pupilometria têm sido utilizados com frequência no contexto educacional. [Lai et al.;2013] aponta que o número de estudos sobre o uso do eye-tracking na aprendizagem cresceu significativamente entre os anos de 2009 e 2012, e que esta técnica pode facilitar o entendimento da relação entre a aprendizagem e os processos cognitivos.

Relacionando esses dados ao aumento da oferta de cursos na modalidade EaD [ABED;2017], cujos recursos pedagógicos são geralmente apresentados no modo audiovisual, comparar a frequência de cada tipo de recurso e identificar na literatura da área os aspectos utilizados para a análise no contexto do rastreio ocular e pupilometria, pode identificar carências e lacunas de pesquisa, assim como direcionar futuras pesquisas.

Nesse sentido, esse mapeamento sistemático tem como objetivo fazer uma investigação estatística dos estudos primários que utilizam rastreio ocular e análise de pupilometria no contexto educacional ou de aprendizagem. Como resultado pretende-se 
VIII Congresso Brasileiro de Informática na Educação (CBIE 2019)

Anais do XXX Simpósio Brasileiro de Informática na Educação (SBIE 2019)

identificar quais os tipos de recursos pedagógicos avaliados, assim como os processos fisiológicos e/ou cognitivos considerados nesses estudos.

\section{Metodologia}

Para a realização desse mapeamento sistemático foi utilizada a metodologia de pesquisa baseada no modelo proposto por [Petersen et al.;2008]. Essa metodologia compreende a definição de questões de pesquisa (Subseção 2.1), a determinação das bases científicas digitais nas quais serão realizadas as buscas (Subseção 2.2), a estruturação de palavraschave que compõem a string de busca (Subseção 2.3), a escolha de critérios de inclusão e de exclusão de artigos no contexto da pesquisa (Subseção 2.4), e a criação de categorias de análise (Subseção 2.5).

\subsection{Definição de Questões de Pesquisa}

Para o presente mapeamento, duas foram as questões de pesquisa propostas:

1.QP01 Quais os tipos de recursos pedagógicos visuais mais analisados com um eye-tracker ou sensor de pupilometria no contexto educacional?

2.QP02 Quais são os aspectos fisiológicos e/ou cognitivos relacionados à aprendizagem observados a partir de dados coletados por um eye-tracker no contexto educacional?

Tais questões foram pensadas com a finalidade de obter um panorama sobre as pesquisas atuais, buscando investigar o que já foi explorado no domínio acadêmico no contexto educacional. A primeira questão pretende fazer uma relação dos recursos pedagógicos visuais examinados em estudos primários, indicando, nos resultados, a frequência de cada um deles. Entende-se por recursos pedagógicos visuais os recursos de aprendizagem que requisitam dos usuários o direcionamento e a atenção do olhar, como, por exemplo, textos, videoaulas, gráficos etc; mesmo que outros sentidos sejam complementares à função dos recursos visuais, como é o caso do áudio na videoaula.

A segunda questão busca entender quais foram os aspectos fisiológicos e/ou cognitivos tidos como parâmetros de análise no conteúdo dos artigos científicos em pauta. Entende-se por aspectos fisiológicos e/ou cognitivos aqueles que se relacionam com o funcionamento intrínseco ao ser humano, como a movimentação dos olhos, dilatação pupilar; ou com processos mentais específicos do cérebro humano ao adquirir conhecimentos, como a percepção, atenção, memória, afetividade, etc.

\subsection{Bases Científicas Digitais}

Foram escolhidas as bases Science Direct e Springer Link por conterem artigos de diversas áreas do conhecimento, incluindo as áreas que abrangem esse mapeamento, sendo assim úteis para o presente trabalho. Além dessas bases, foram escolhidas bases especializadas em informática no contexto educacional brasileiro, que são: os anais do Workshop de Informática na Escola (WIE) e do Simpósio Brasileiro de Informática na Escola (SBIE), assim como as publicações na Revista Brasileira de Informática na Educação (RBIE).

\subsection{Estruturação de Palavras-Chave}

Seguindo o modelo de [Petersen et al., 2008], a estruturação de palavras-chave dá-se pela pesquisa manual de strings nas bases supracitadas a fim de estabelecer uma cadeia 
VIII Congresso Brasileiro de Informática na Educação (CBIE 2019)

Anais do XXX Simpósio Brasileiro de Informática na Educação (SBIE 2019)

de strings que compreenda o maior número de artigos e contribua para a solução das questões de pesquisa.

Após pesquisas realizadas com combinações diferentes de cadeia de strings, a combinação mais satisfatória é apresentada na Tabela 1, discriminadas por áreas de interesse. Cada base digital possui seu próprio sistema e formatação de busca, portanto, no processo de pesquisa, foram feitas adequações quanto a formatação levando em consideração essas diferenças.

Após a definição da cadeia de strings, uma busca automática foi realizada, adequando-as aos sistemas e formatações de busca de cada base, retornando 3041 artigos correspondentes para a base Springer Link e 90 para Science Direct. Já para as bases WIE, SBIE e RBIE, foram realizadas pesquisas manuais, com as strings: movimento ocular, movimento dos olhos, eye-tracking, eye-tracker, gaze, pupila $\mathrm{e}$ pupilometria. Apenas um artigo foi encontrado com essa pesquisa, mas não correspondia ao objetivo desse mapeamento por se encaixar nos critérios de exclusão.

Tabela 1 - Strings utilizadas para pesquisa nas bases científicas digitais

\begin{tabular}{|c|}
\hline Strings relativas à educação: \\
("education" OR "multimedia learning" OR "e-learning") \\
\hline$A N D$ \\
\hline $\begin{array}{c}\text { Strings relativas ao movimento ocular ou dilatação da pupila: } \\
\text { ("eye-tracking" OR "eye gaze" OR "pupil size" OR "pupilometry" OR "pupil dilatation") }\end{array}$ \\
\hline
\end{tabular}

\subsection{Critérios de Inclusão e de Exclusão}

A Tabela 2 apresenta os critérios de inclusão e de exclusão utilizados.

Tabela 2 - Critérios de inclusão e de exclusão

\begin{tabular}{|c|c|}
\hline Critérios de Inclusão & Critérios de Exclusão \\
\hline $\begin{array}{l}\text { 1. Estudos que utilizem um eye-tracker ou um } \\
\text { instrumento medidor da dilatação da pupila com } \\
\text { aplicação no contexto educacional } \\
\text { 2. Estudos que coletem e analisem dados } \\
\text { experimentais no uso de recursos pedagógicos }\end{array}$ & 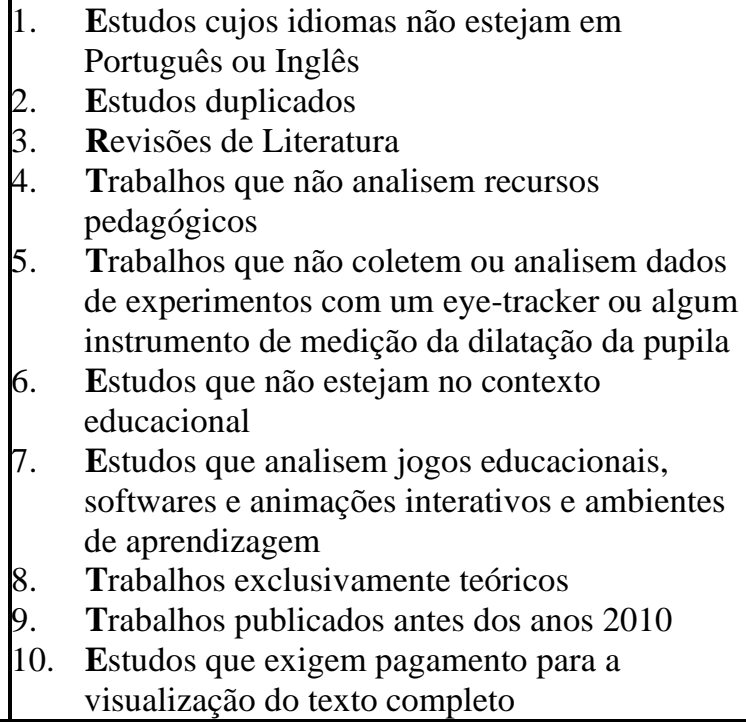 \\
\hline
\end{tabular}

A escolha por excluir documentos que analisem jogos educacionais, softwares e animações interativas e ambientes de aprendizagem deu-se porque essas são tecnologias mais complexas, com vários metadados que necessitam de estudos mais detalhados sobre a ampla gama de propriedades concernentes. 
VIII Congresso Brasileiro de Informática na Educação (CBIE 2019)

Anais do XXX Simpósio Brasileiro de Informática na Educação (SBIE 2019)

\subsection{Categorias de Análise}

As categorias de análise são os métodos de classificação usados para fins de análise estatística dos documentos encontrados nas bases de dados digitais investigadas. No presente estudo, porém, essas categorias, em si mesmas, respondem parcialmente às questões de pesquisa. São elas: recursos pedagógicos visuais e aspectos fisiológicos e/ou cognitivos. Sendo assim, sempre que uma nova subcategoria fosse observada, era incluída na lista de categorias pertinente. As subcategorias encontradas relativas aos recursos pedagógicos visuais foram: texto, imagem, apresentação em forma de slides, vídeo, diagrama e gráfico. Já as relativas aos aspectos fisiológicos e/ou cognitivos foram: movimento ocular, atenção, processos, afetividade, esforço cognitivo e dilatação pupilar. Cada subcategoria encontrada é apresentada e definida detalhadamente na Seção 3.

Além dessas, foram criadas classificações referentes à população experimental e ao processo pedagógico em questão. São elas: população de estudo e contexto educacional. A categoria população de estudo foi subdividida em: educação infantil(Estudantes da educação infantil e/ou crianças de até 5 anos de idade, assim como redação dada pela Lei $\mathrm{n}^{\circ} 12.796$, de 2013); fundamental (estudantes do ensino fundamental), médio(estudantes do ensino médio), superior(estudantes de graduação, pós-graduação ou pessoas já graduadas) e outros(não estudantes ou não informado pelo documento). A categoria contexto educacional foi subdividida em: aprendizagem e avaliação. Na aprendizagem, são avaliados os processos de aquisição de conhecimento, apresentado após experimentação, testes de retenção e aprendizagem. Na avaliação são investigados os processos avaliativos que podem ou não contar com o conhecimento prévio do experimentado.

\subsection{Triagem dos Artigos}

Após o término das buscas, o próximo passo é a investigação dos 3131 artigos correspondentes à busca por cadeia de strings. Durante a própria busca foi possível aplicar os critérios de exclusão 9 e 10 a partir de filtros próprios da busca automática.

A investigação foi conduzida em três etapas. A primeira corresponde a uma leitura dos títulos, dos resumos e das palavras-chave dos artigos. Através dessa leitura, foi possível aplicar os critérios de exclusão 1, 2, 3, 7 e 8 - já que não é necessária uma leitura profunda para identificar esses aspectos. Nessa etapa, 2916 artigos foram excluídos da pesquisa. A segunda etapa corresponde à verificação dos 215 artigos restantes quanto aos critérios de exclusão 2, 4, 5 e 6. Para isso, foi feita a leitura da maior parte do conteúdo dos documentos, principalmente os tópicos relacionados à metodologia e a conclusão. Nessa etapa, 84 artigos foram excluídos da pesquisa. $\mathrm{Na}$ última etapa, foram analisados os 131 artigos restantes, buscando identificar quais não se enquadram nos critérios de inclusão. Após essa análise 121 artigos foram selecionados, sendo um deles um livro que continha dois experimentos diferentes. Cada experimento foi considerado um documento distinto para uma análise mais detalhada das categorias e subcategorias. Todo o processo de pesquisa resultou em 122 documentos para uma análise detalhada.

\section{Resultados e Discussão}


VIII Congresso Brasileiro de Informática na Educação (CBIE 2019)

Anais do XXX Simpósio Brasileiro de Informática na Educação (SBIE 2019)

A partir da análise dos dados do mapeamento sistemático foi possível extrair informações sobre o cenário atual de pesquisa no contexto visado, que poderão auxiliar o entendimento de quais áreas necessitam ser mais exploradas em estudos futuros.

Para responder às questões de pesquisa foi necessário definir subcategorias para as categorias: recursos pedagógicos visuais e aspectos fisiológicos e/ou cognitivos, já mencionadas anteriormente (Tabela 3).

Tabela 3 - Tipos de recursos pedagógicos visuais encontrados

\begin{tabular}{|l|l|}
\hline Tipo de Recurso & \multicolumn{1}{|c|}{ Definição } \\
\hline Textos & $\begin{array}{l}\text { Documentos impressos ou virtuais que utilizem conjuntos de palavras, números ou } \\
\text { ideogramas para expressar um significado. Expressões matemáticas também foram incluídas } \\
\text { nesta subcategoria. }\end{array}$ \\
\hline .Imagens & $\begin{array}{l}\text { Representações visuais de pessoas, objetos, figuras geométricas, cenas, animais, etc em } \\
\text { forma de ilustrações, sejam elas impressas ou virtuais. }\end{array}$ \\
\hline $\begin{array}{l}\text { Apresentações } \\
\text { em forma de } \\
\text { slides }\end{array}$ & $\begin{array}{l}\text { Sequências de telas coerentes dentro de um contexto. Nos artigos analisados, grande parte } \\
\text { das apresentações é composta de textos e imagens. }\end{array}$ \\
\hline Vídeos & $\begin{array}{l}\text { Representam sequências de imagens em movimento que tem objetivo de instruir quem } \\
\text { fazendo uso do mesmo para uma dada finalidade, podendo fazer uso de lousas, instrutores e } \\
\text { representações de cenas. }\end{array}$ \\
\hline Diagramas & $\begin{array}{l}\text { Representações gráficas para demonstrar e auxiliar o entendimento em um esquema } \\
\text { simplificado. Imagens com rótulos que demonstrem a localização ou função de partes } \\
\text { contidas em um sistema maior também foram consideradas como diagramas. }\end{array}$ \\
\hline Gráfico & $\begin{array}{l}\text { Representações visuais que visam a apresentação de dados podendo ou não estarem } \\
\text { dispostas em eixos. }\end{array}$ \\
\hline
\end{tabular}

Já para a categoria relativa aos aspectos fisiológicos e/ou cognitivos, foram observadas seis subcategorias (Tabela 4) detalhadas a seguir:

Tabela 4 - Tipos de aspectos fisiológicos e/ou cognitivos encontrados

\begin{tabular}{|l|l|}
\hline \multicolumn{1}{|c|}{$\begin{array}{c}\text { Tipo de } \\
\text { Recurso }\end{array}$} & \multicolumn{1}{|c|}{ Definição } \\
\hline $\begin{array}{l}\text { Movimento } \\
\text { ocular }\end{array}$ & $\begin{array}{l}\text { Identifica os locais onde há o direcionamento do olhar, buscando justificativas e padrões para } \\
\text { tal direcionamento. }\end{array}$ \\
\hline Atenção & $\begin{array}{l}\text { Percebe onde está o foco do olhar através do tempo de fixação, ou seja, na duração em que o } \\
\text { foco estava direcionado a certa área pré-definida. }\end{array}$ \\
\hline Processos & $\begin{array}{l}\text { Define interesse nos elementos relativos ao processo cognitivo, como os diferentes tipos } \\
\text { memória, conhecimento prévio, estratégias de aprendizagem, estado de ânimo do } \\
\text { experimentado dadas certas circunstâncias (motivação) etc. }\end{array}$ \\
\hline Afetividade & Está interessada nos estados afetivos relacionados à emoção. \\
\hline $\begin{array}{l}\text { Esforço } \\
\text { Cognitivo }\end{array}$ & Define interesse pela análise de carga cognitiva ou esforço mental exercidos em uma ação. \\
\hline Dilatação Pupilar & Define interesse pela variação da pupila dos participantes dos experimentos. \\
\hline
\end{tabular}

Foi possível observar também que diversas combinações entre as subcategorias dos recursos pedagógicos visuais e aspectos fisiológicos e/ou cognitivos foram analisadas, como, por exemplo, o uso de texto com imagens, texto com diagramas, slides e vídeos, movimento ocular com atenção, atenção e processos, etc.

Identificadas e detalhadas as subcategorias, as questões de pesquisa podem ser respondidas. A questão de pesquisa 01 (QP1) identifica os tipos de recursos pedagógicos visuais. O texto foi o recurso pedagógico visual mais investigado em pesquisas, com um número total de 49 trabalhos em que este recurso foi o foco central. O Gráfico 1 apresenta a quantidade de uso dos diferentes recursos e suas combinações. 
VIII Congresso Brasileiro de Informática na Educação (CBIE 2019)

Anais do XXX Simpósio Brasileiro de Informática na Educação (SBIE 2019)

\section{Gráfico 1 - Análise de Recursos Pedagógicos Visuais}

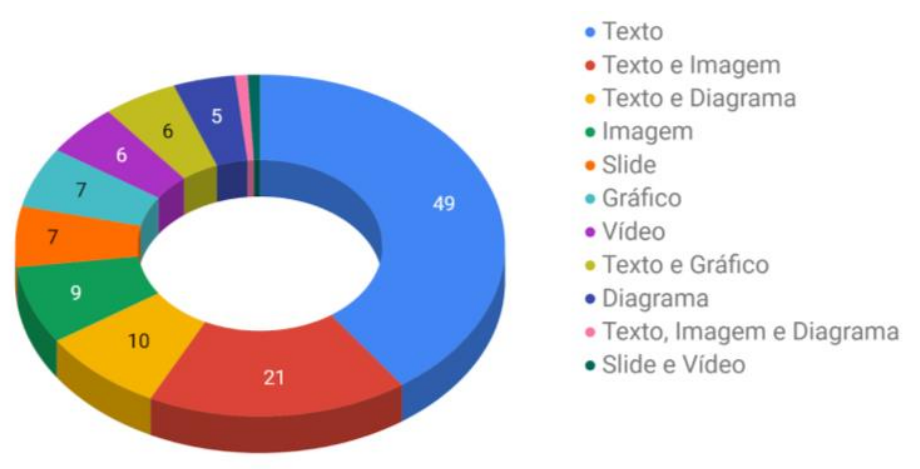

Para outro modo de investigação, a Tabela 5 apresenta os dados de forma a contabilizar os recursos pedagógicos visuais tanto em publicações que os analisaram individualmente quanto aos que combinaram outros recursos em sua análise.

Tabela 5 - Análises Individualizadas de Recursos Pedagógicos Visuais

\begin{tabular}{|c|c|c|}
\hline Recursos Pedagógicos Visuais & Análises Individuais & $\begin{array}{c}\text { Análises individuais e em } \\
\text { combinações }\end{array}$ \\
\hline Texto & 49 & 87 \\
\hline Imagem & 9 & 31 \\
\hline Diagrama & 5 & 16 \\
\hline Gráfico & 7 & 13 \\
\hline Slide & 7 & 8 \\
\hline Vídeo & 6 & 7 \\
\hline
\end{tabular}

Contabilizando suas análises individuais e combinações com outros recursos, o texto configura o recurso pedagógico visual investigado com mais frequência, totalizando 87 artigos, aproximadamente $71 \%$ de um conjunto de 122 artigos. Ademais, pode-se destacar que o vídeo, seja ele instrucional ou em forma de vídeoaula, foi pouco explorado em pesquisas científicas no contexto do mapeamento, sendo investigado apenas em 7 estudos.

A segunda questão de pesquisa (QP2) investiga quais os aspectos fisiológicos e/ou cognitivos relacionados à aprendizagem são observados a partir de dados coletados com um eye-tracker. Dos 122 artigos selecionados pela metodologia descrita, muitos desses combinam diferentes tipos de recursos pedagógicos visuais em um mesmo material utilizado nos experimentos. No Gráfico 2, as combinações que foram analisadas em apenas um artigo foram agrupadas na subcategoria Outros ${ }^{1}$.

$\mathrm{Na}$ Tabela 6 encontram-se dados referentes às subcategorias relacionadas ao número de vezes em que foram analisadas, tanto de formas individuais, como contabilizando suas combinações com outros aspectos. As subcategorias Dilatação Pupilar, Processos e Afetividade não apresentaram análises individuais específicas, pois não podem ser analisadas sem relacioná-las a outro aspecto. Assim, a questão de

\footnotetext{
1 Dilatação Pupilar, Esforço Cognitivo e Afetividade (1), Mov. Ocular, Atenção e Afetividade (2), Mov. Ocular, Atenção e Afetividade e Esforço Cognitivo (3), Mov. Ocular, Esforço Cognitivo e Dilatação Pupilar (4), Atenção e Esforço Cognitivo (5), Mov. Ocular, Atenção e Esforço Cognitivo (6), Mov. Ocular, Atenção, Esforço Cognitivo, Processos e Dilatação Pupilar (7), Mov. Ocular, Atenção, Esforço Cognitivo e Dilatação Pupilar (8).
} 
VIII Congresso Brasileiro de Informática na Educação (CBIE 2019)

Anais do XXX Simpósio Brasileiro de Informática na Educação (SBIE 2019)

pesquisa 2 pode ser respondida tanto pela identificação de tais aspectos como o uso combinado dos mesmos

\section{Gráfico 2 - Análise de Aspectos Fisiológicos e/ou Cognitivos}

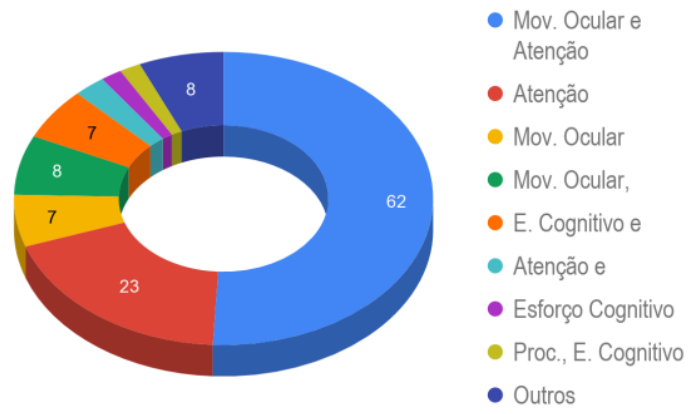

Tabela 6 - Análises Individualizadas de Aspectos Fisiológicos e/ou Cognitivos

\begin{tabular}{|c|c|c|}
\hline $\begin{array}{c}\text { Aspectos Fisiológicos e/ou } \\
\text { Cognitivos }\end{array}$ & Análises Individuais & $\begin{array}{c}\text { Análises Individuais e } \\
\text { Combinações }\end{array}$ \\
\hline Atenção & 23 & 102 \\
\hline Mov. Ocular & 8 & 83 \\
\hline Esforço Cognitivo & 2 & 18 \\
\hline Processos & 0 & 14 \\
\hline Dilatação Pupilar & 0 & 13 \\
\hline Afetividade & 0 & 3 \\
\hline
\end{tabular}

A partir da Tabela 6 é possível observar que os artigos das bases científicas pesquisadas tendem a explorar mais a atenção e os padrões de comportamento do movimento ocular. De forma geral, os artigos que analisaram esses dois aspectos mencionados a partir de apenas um recurso pedagógico, tem como objetivo ou encontrar padrões nas estratégias de uso dos mesmos, como, por exemplo, comparando estratégias de aprendizagem entre aprendizes e especialistas [Barnes, A., 2016; Broadbent, M., 2013; Dogusoy-Taylan, B., 2014; Kok, E., 2015; Liu, H., 2015; Panse, A., 2012]. Além disso, buscam também comparar diferentes formas de apresentação de um mesmo recurso, verificando a influência, por exemplo, de diferentes tipos de fontes e cores e também das diferenças entre os formatos impresso e digital [Bochud, Y., 2013; Chen, S., 2015; Johnston, N., 2016; Dogusoy, B., 2016; Rello, L., 2015; White, S., 2011].

Em geral, o foco dos trabalhos que envolviam a análise de dois ou mais recursos pedagógicos visuais foi investigar a influência de um dos recursos quando usados em conjunto com outro, por exemplo, o artigo de [Jian, Y., 2014] que investiga a influência de diagramas no entendimento de um texto científico expositório, bem como as estratégias na associação dos dois recursos cujos resultados relaciona um pior desempenho em testes de retenção a alunos que somente leram o texto.

O Diagrama 1 apresenta os resultados do mapeamento sistemático realizado de modo a obter um panorama para comparação entre as categorias que respondem as questões de pesquisa, a população e o contexto de aprendizagem nos quais foram implementados. Para a realização desse diagrama, foram considerados os recursos pedagógicos e os aspectos fisiológicos e/ou cognitivos como individuais e suas combinações, ou seja, para cada combinação na análise de dois ou mais atributos, uma unidade é adicionada a cada um. 


\section{Diagrama 1 - Panorama da distribuição dos artigos selecionados por categorias}

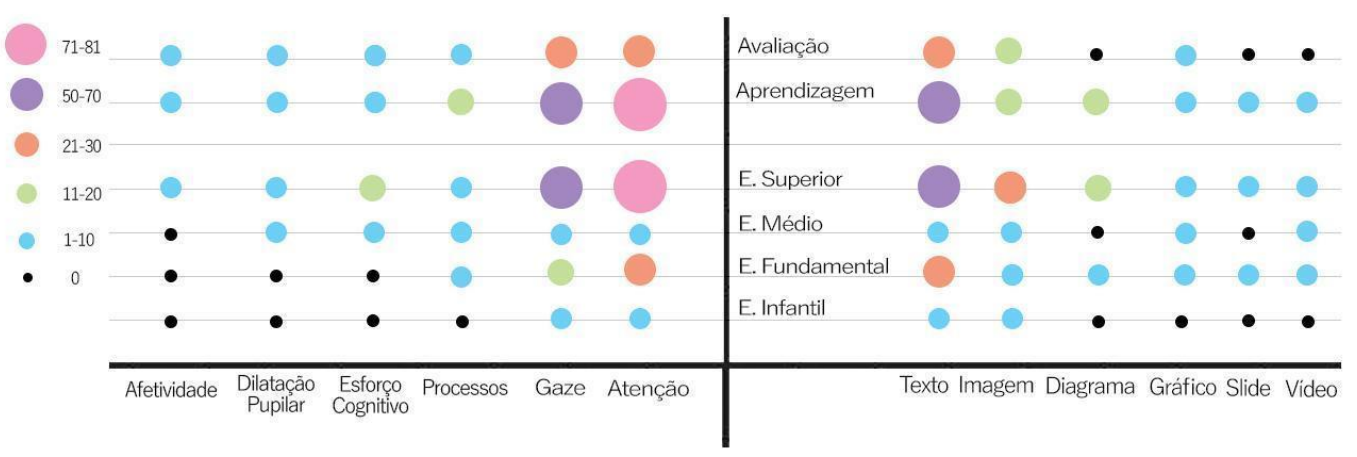

A partir do Diagrama 1, podem ser observados campos de pesquisa carentes de mais estudos, representados pelos pontos menores e mais escuros, os quais não apresentaram nenhum resultado na pesquisa realizada. A subcategoria que menos apresentou resultados nas buscas foi a afetividade. Em estudos sobre neurociência e educação, [Goswami, U., 2004] relaciona estudos que relacionam a afetividade e cognição, indicando que diferentes estados afetivos podem influenciar a cognição. Logo, a associação de dados de eye-tracking e de pupilometria com a análise de estados afetivos poderiam beneficiar a comunidade escolar.

A educação infantil foi a população que apresentou o menor número de estudos dentre todas as outras populações. Uma possível causa para tal deve-se às dificuldades envolvidas em pesquisas com crianças mais novas e bebês, que envolvem $o$ consentimento e disponibilidade dos responsáveis e a problemática ética dessas pesquisas. Além disso, em geral, o número de estudos que investigam avaliações no contexto pesquisado é muito menor comparado aos estudos que envolvem a aquisição de aprendizagem, apontando uma carência principalmente no uso de diagramas em avaliações, o qual não apresentou nenhum resultado, embora haja bastante uso no âmbito educacional.

\section{Ameaças à Validade}

As questões de pesquisa e os critérios de inclusão e exclusão foram definidos antes da execução do mapeamento, exceto a janela de tempo escolhida nos critérios de exclusão. As ameaças à validade se configuram no mecanismo de busca das bases digitais e dos anais dos eventos e na escolha de um número restrito de bases científicas digitais, o que pode fazer com que estudos relevantes não estejam presentes nesse mapeamento. Além disso, dada a quantidade de artigos analisados, equívocos relacionados às condições humanas na seleção e classificação não podem ser desconsiderados.

\section{Conclusão}

O principal objetivo desse mapeamento sistemático foi o de obter um panorama do cenário atual de pesquisa relacionado à aplicação da técnica de rastreio ocular no contexto da educação e da aprendizagem. Em síntese, os resultados apresentados e discutidos na seção 3 apontam o texto como recurso pedagógico visual mais investigado, em contraste com o vídeo, com percentuais do total de artigos de $71 \%$ e $6 \%$ respectivamente. Vale ressaltar que, de acordo com o censo realizado em 2017 pela Associação Brasileira de Educação a Distância [ABED;2017], no período de 2009 a 2017, o volume de matrículas em cursos a distância e semipresenciais aumentou em 
aproximadamente $14.714 \%$. Além disso, dentre as instituições pesquisadas, $34 \%$ oferecem espaço dedicado em seus pólos para a transmissão de videoaulas, o que permite observar que pelo menos $34 \%$ das instituições fazem uso das videoaulas como ferramenta pedagógica. Portanto, novas pesquisas sobre o uso dessa ferramenta trariam muitos benefícios à educação a distância. Dos aspectos fisiológicos e/ou cognitivos, a atenção (44\%) foi o aspecto mais pesquisado e o menos observado foi a afetividade (1\%), o que sugere que há ainda espaço para investigação.

Assim, a partir deste mapeamento é possível observar lacunas de pesquisa e direcionar estudos futuros para preencher os espaços em que há a necessidade de maior investigação, beneficiando todo o domínio da educação e da aprendizagem.

\section{Agradecimentos}

O presente trabalho é parte de um projeto de Iniciação Científica, modalidade Pesquisando Desde o Primeiro Dia (PDPD), que teve como objetivo explorar os dados de dilatação de pupila através de um dispositivo eye-tracker em sessões de videoaulas. Os autores agradecem à Pró-reitoria de pesquisa da UFABC pela bolsa concedida.

\section{Referências}

ABED Censo EAD.BR: relatório analítico da aprendizagem a distância no Brasil 2017 $=$ Censo EAD.BR: analytic report of distance learning in Brazil 2017 [livro eletrônico]/[organização] ABED - Associação Brasileira de Educação a Distância; [traduzido por Maria Thereza Moss de Abreu]. Curitiba: InterSaberes, 2018.

Barnes, Adrienne E., and Young-Suk Kim. "Low-Skilled Adult Readers Look like Typically Developing Child Readers: a Comparison of Reading Skills and Eye Movement Behavior.” Reading and Writing, vol. 29, no. 9, 2016, pp. 1889-1914.

Bochud, Yves Etienne, and Marc Garbely. "Reading on EInk and Backlit LED - The Influence of Positive and Negative Contrast on Eye Movements." Lecture Notes in Computer Science Human Factors in Computing and Informatics, 2013, pp. 711720.

Broadbent, Marc, et al. "Comparing Novice and Expert Nurses in Analysing Electrocardiographs (ECGs) Containing Critical Diagnostic Information: An Eye Tracking Study of the Development of Complex Nursing Visual Cognitive Skills." Current Trends in Eye Tracking Research, 2013, pp. 297-315.

Chen, Sheng-Chang, et al. "The Effects of Static versus Dynamic 3D Representations on 10th Grade Students' Atomic Orbital Mental Model Construction: Evidence from Eye Movement Behaviors." Computers in Human Behavior, vol. 53, 2015, pp. 169180.

Dogusoy, Berrin, et al. "How Serif and Sans Serif Typefaces Influence Reading on Screen: An Eye Tracking Study." Design, User Experience, and Usability: Novel User Experiences Lecture Notes in Computer Science, 2016, pp. 578-586.

Dogusoy-Taylan, Berrin, and Kursat Cagiltay. "Cognitive Analysis of Experts' and Novices' Concept Mapping Processes: An Eye Tracking Study." Computers in Human Behavior, vol. 36, 2014, pp. 82-93. 
VIII Congresso Brasileiro de Informática na Educação (CBIE 2019)

Anais do XXX Simpósio Brasileiro de Informática na Educação (SBIE 2019)

Goswami, U.. Neuroscience and education. British Journal of Educational Psychology, 74: 1-14, 2004

Jian, Yu-Cin, and Chao-Jung Wu. "Using Eye Tracking to Investigate Semantic and Spatial Representations of Scientific Diagrams During Text-Diagram Integration." Journal of Science Education and Technology, vol. 24, no. 1, 2014, pp. 43-55.

Johnston, Nicole, et al. "Print and Digital Reading Preferences and Behaviors of University Students in Qatar." Information Literacy: Key to an Inclusive Society Communications in Computer and Information Science, 2016, pp. 247-255.

${ }^{1}$ Kok, Ellen M., et al. "Case Comparisons.” Academic Radiology, vol. 22, no. 10, 2015, pp. 1226-1235., doi:10.1016/j.acra.2015.04.012.

${ }^{2}$ Kok, Ellen M., et al. "Systematic Viewing in Radiology: Seeing More, Missing Less?" Advances in Health Sciences Education, vol. 21, no. 1, 2015, pp. 189-205.

Liu, Han-Chin, et al. "Using Eye-Tracking Technology to Investigate the Impact of Different Types of Advance Organizers on Viewers' Reading of Web-Based Content: A Pilot Study." Advances in Intelligent and Soft Computing Advanced Information Technology in Education, 2012, pp. 63-69.

Mafra, S. N.; Travassos, G. H. Estudos Primários e Secundários apoiando a busca por Evidências em Engenharia de Software; Rio de Janeiro: PESC-COPPE/UFRJ 2006.

Meng-Lung Lai, Meng-Jung Tsai, Fang-Ying Yang, Chung-Yuan Hsu, Tzu-Chien Liu, Silvia Wen-Yu Lee, Min-Hsien Lee, Guo-Li Chiou, Jyh-Chong Liang, Chin-Chung Tsai. A review of using eye-tracking technology in exploring learning from 2000 to 2012, Educational Research Review, Volume 10, 2013, Pages 90-115, ISSN 1747938X,

Panse, Anja, et al. "Reading Proofs for Validation and Comprehension: an ExpertNovice Eye-Movement Study." International Journal of Research in Undergraduate Mathematics Education, vol. 4, no. 3, 2018, pp. 357-375. Petersen, K., Feldt, R., Mujtaba, S., Mattsson, R.. Systematic mapping studies in software engineering. In 12th International Conference on Evaluation and Assessment in Software Engineering, 17: 71-80, 2008.

Rello, Luz, and Ricardo Baeza-Yates. "How to Present More Readable Text for People with Dyslexia." Universal Access in the Information Society, vol. 16, no. 1, 2015, pp. 29-49.

White, Sarah J., et al. "Eye Movement Behaviour during Reading of Japanese Sentences: Effects of Word Length and Visual Complexity." Reading and Writing, vol. 25, no. 5, 2011, pp. 981-1006. 